(C) 2011 IEEE. Personal use of this material is permitted. Permission from IEEE must be obtained for all other uses, in any current or future media, including reprinting/republishing this material for advertising or promotional purposes, creating new collective works, for resale or redistribution to servers or lists, or reuse of any copyrighted component of this work in other works. 


\title{
Model Predictive Control of Murray-Darling Basin Networks
}

\author{
L. Li, N. Okello, M. Pham, S. K. Saleem, W. Qiu, R. Evans, I. Mareels
}

\begin{abstract}
River basins are the most significant component in water supply grids and are under increasing pressure from competing demands for fresh water. However, unlike energy grids which are managed very efficiently using closed-loop operation, water grids, and river basins in particular, are largely open-loop systems. One reason is the difficulty associated with developing suitable models and feedback controllers. This paper proposes a systematic approach using model predictive control based on simple low order models for the real-time operation of entire river basin networks.

Index Terms-River basin, Real-time control, Model predic-
\end{abstract} tive control.

\section{INTRODUCTION}

River basins are water distribution networks made up of natural water carriers. Examples include the Amazon, Ganges, Mississippi, and Congo Basins. These system are composed of rivers, stream, lakes, estuaries, groundwater reservoirs, wetlands, etc. River basins also include the land surfaces that channel water into the carriers. Since irrigation was first conceived in the 6th millennium BC in Mesopotamia, river basins throughout the world have been enhanced and exploited through the construction of control points including dams, weirs, locks, canals, etc. It is interesting to note that the general operation of these systems has not changed much since then. Water is still collected and stored at altitude. When required, it is released from dams and transported downstream under the influence of gravity and routed through a network of rivers and canals [Mar09]. This apparently simple process is affected by a diminishing supply, unpredictable timing of demand, and an increasingly complex set of competing objectives (e.g. ecological, agricultural, and consumptive use). The lack of accurate short to medium term supply and demand forecasts and the lack of a systematic framework for balancing operational objectives are typical of river basins. Whilst addressing these issues will not solve the so-called global water crisis, they represent an important pre-requisite to a total water security solution. A systematic approach based on feedback control offers one

National ICT Australia is funded by the Australian Governments Department of Communications, Information Technology, and the Arts and the Australian Research Council through Backing Australias Ability and the ICT Research Centre of Excellence programs.

L. $\mathrm{Li}$ is with University of Technology, Sydney 1i.1i@uts.edu.au. This work was done when Dr. L. Li was with National ICT Australia Ltd.

\{N. Okello, M. Pham, S. K. Saleem, W. Qiu, R. Evans are with National ICT Australia Ltd \{nickens.okello, minh.pham, khusro.saleem, wanzhi.qiu rob.evans\}@nicta.com.au

I. Mareels is with The University of Melbourne, Australia iven.mareels@unimelb.edu.au solution to real-time river operation, and is the subject of this paper.

Real-time river basin operation is typical of large-scale control problems that have the following characteristics [Pap84]:

1) A network structure with some kind of flow along links connecting storage units;

2) Flow is to be routed from specific sources to designated destinations;

3) Flow is subject to capacity constraints;

4) There are time-varying demand variables at the source, along the network and at the destination;

5) The links and storage units are characterized by transport lags; and,

6) A communication network with limited bandwidth is used to transmit network state.

In a river network, gate flows are control variables while storage volumes and flows along the river are the controlled variables. Inflows into the network come from tributaries, rainstorms and groundwater reservoirs, and losses can occur due to evapotranspiration and seepage to underground reservoirs. These inflows and losses whose values may be obtained through estimates and forecasts are categorized under disturbances. The loads in a river network are consumptive extractions by the water authorities operating within the basin and can be estimated using demand forecast. The objective is to specify control inputs that influence storages and flows within the network such that for any level disturbance within the network, performance criterion is minimized subject to capacity constraints and time varying loads.

While flow and storage levels are the major quantitative values of interest, other variables $e, g$., salinity, hardness, alkalinity, concentration of salts of strong acids, permanganate values, chemical oxygen demand, biochemical oxygen demand (BOD), total organic carbon (TOC), etc, associated with water quality are also important. If they can be measured and modeled, then they can also be controlled jointly with the quantitative variables.

Modeling and optimization of water resources systems has a rich history [Lab04]. The Saint-Venant equations [Cho88] are the basis for the mathematical modeling of open water channels. These are hyperbolic partial differential equations making them difficult to use in feedback controller design. The study in [Wey02] has focused on the use of decentralized PI control, and [LF06] used $H_{\infty}$ control. The studies in [NvOKdS09] explored the use of model predictive control. An alternative to the Saint-Venant equations is to exploit grey-box or data-based models derived using system identification experiments by [JH93], [You98]. The key advantage 
of these models is that feedback controllers are easier to design $\left[\mathrm{MWO}^{+} 05\right]$.

Most of the studies cited above focus on modeling and control of irrigation canal networks and short river reaches. Combined simulation-optimization methods are commonly used to plan and operate river basin networks [BB09]. This paper builds on previous work in open canals to develop a framework for real-time river basin operation based on model predictive control theory. The River Murray system in Australia is used as a case study.

\section{A. Murray-Darling basin networks}

The River Murray system [MDB06a] drains a catchment region which covers the south east corner of the Australian continent and extends over $1,060,000 \mathrm{~km}^{2}$. The total length of the main river channel is $3,780 \mathrm{~km}$ and the mean discharge is $0.4 \mathrm{ML} / \mathrm{sec}$. The system is largely fed by precipitation and snow-melt in the Australian Alps. The main consumptive demands are irrigation districts and rural populations and one major metropolitan demand site in Adelaide, South Australia. The River Murray is permanently navigable to a distance of 970 kilometers from the mouth due to a series of locks and weirs.

The River Murray is operated in three modes [MDB06b]: (1) Supplying mode; (2) Storing mode; and (3) Spilling mode. It is possible for different reaches of the river to be in different modes. Supplying mode occurs during the irrigation season. The flow in the river is set to meet demands with little excess. Storing mode generally occurs when the flows in the river are in excess of that required to meet diversions, water supply, and minimum flow requirements; but which are confined within the channel. Spilling mode occurs when flow exceeds the river's channel capacity at a point as a result of runoff generated by heavy rain. This operation can be quite complex as the flow varies as tributaries join the main stream.

\section{B. Control objectives}

Controlling a river basin network involves regulating a selected set of states around their set-points based on the operational mode of interest. For example in storage mode a river operator maintains water levels in storages at specified levels while allowing flows to take on values necessary to maintain those levels. On the other hand, in supply mode a river operator maintains constant flow rates in-stream while allowing storage levels to take on values necessary to maintain those flows. This section summarizes the main operational objectives for the River Murray [MDB06a]:

1) Meet water demands for both consumptive use and environmental flows, expressed as a flow rate (set-point regulation);

2) Keep storage water levels close to a reference level (also set-point regulation);

3) Reject disturbances caused by urban and irrigation withdrawals and rainfall-runoff;

4) Minimize control effort by minimizing gate movement; and,
5) Maintain rate of rise and rate of fall within bounds to avoid river bank slumping.

\section{Model predictive control}

Model predictive control (MPC) [Mac02], [MRRS00] is one of the leading advanced control technologies in the process industries. The most attractive feature of MPC is the ability to accommodate complex performance objectives, dynamic systems and constraints in a unified framework. Similar to the process industries, the dynamics of water systems are relatively slow. Also, during control design, physical limitations and managing water level and flow within certain bounds need to be considered. MPC is a suitable controller design strategy for the current problem. Applications of MPC to water systems can be found in [vO06], [NvOKdS09], [BWdMB08]. This paper proposes a systematic approach using model predictive control based on simple low order models for the real-time operation of entire river basin networks. Relevant work can also be found in $\left[\mathrm{ELM}^{+} 11\right]$.

\section{MODELS}

A schematic of the River Murray System is illustrated in Figure 1. Following the methodology in [Pap84] the river system is subdivided into a sub-networks with storage capabilities. Links connecting the sub-networks are treated as pure delays. In this sense flow rates leaving a subnetwork (or storage element) are control variables, whereas the volumes (or water levels) in the storage elements are the state variables. In-stream flow rates further downstream from storage outlets can also be considered as state variables.

\section{A. Physical models}

1) Storage models: River basin storages are modeled using the continuity equation

$$
\dot{V}(t)=\sum_{n \in I} q_{i n, n}(t)-\sum_{m \in O} u_{m}(t) .
$$

where $V$ is the storage volume, $q_{i n, n}(t)$ and $u_{m}(t)$ are inflow rate and outflow rates respectively, and $I$ and $O$ denote the set of all inflows and outflows, respectively. The inflow is a measurement some distance upstream of the storage. The outflow is defined as a control variable. This approach was originally proposed in [WM73] and later in [Wey01] where the control components are defined in terms of flows.

In river basin operations, storage volume is generally inferred from water level measured at the downstream end of a storage element, close to the outflow control point. The function relating storage volume and water level depends on the storage element's geometry. Assuming only a single outflow structure is present, the following model for water level in a storage element can be used

$$
\dot{y}(t)=\alpha(y)\left(\sum_{n \in I} q_{i n, n}(t)-\sum_{m \in O} u_{m}(t)\right)
$$

where the function $\alpha(y)$ is related to the storage element's geometry. Generally it will be non-linear, for example when 


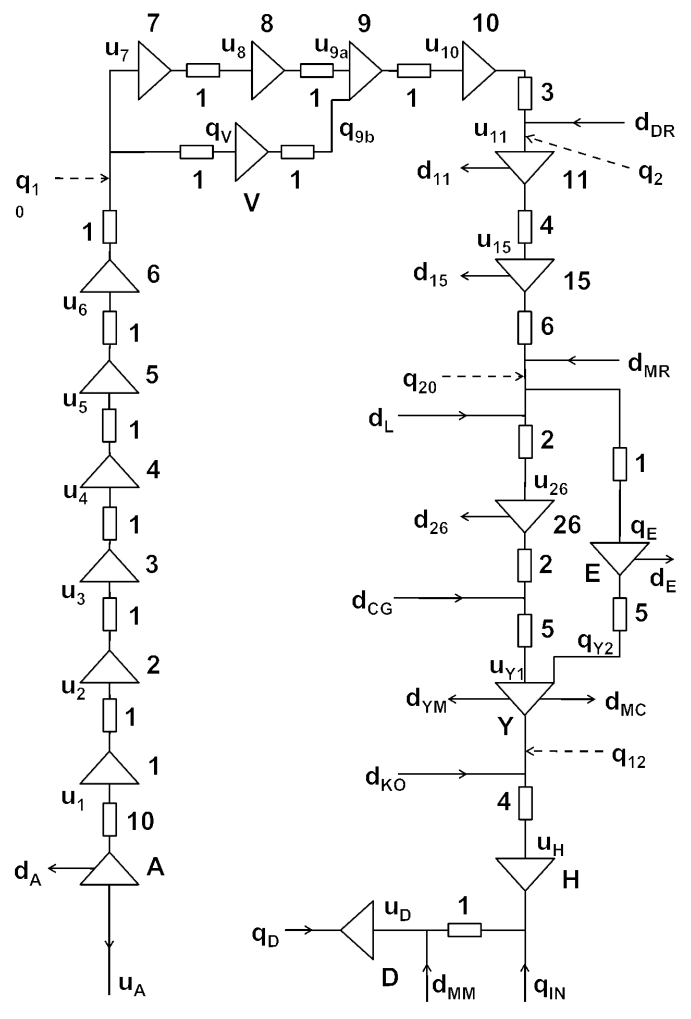

Fig. 1. River Murray schematic

the storage is deep and has sloping sides. In this paper, this value is assumed constant. Without this simplification, the system can still be described by a set of linearized models by selecting several operating points over the range of set-points. Gain scheduling is a popular method used for designing controllers for such systems [SA90]. Letting $T_{s}$ denote the sample interval, and using a first order approximation for $\dot{y}$, the discrete-time model for water level is given by

$$
y[k+1]=y[k]+T_{s} \alpha\left(\sum_{n \in I} q_{i n, n}[k]-\sum_{m \in O} u_{m}[k]\right) .
$$

2) River reach models: The Saint-Venant equations are a good starting point for modeling river reaches. It has been shown in [LG99] (see also [Wey01]) that under relatively mild assumptions, the Saint-Venant equations can be linearlized about a reference flow rate resulting in the following river reach dynamics

$$
\dot{q}_{\text {out }, i}+\frac{1}{K} q_{\text {out }, i}=q_{\text {in }, i}\left(t-\tau_{i}\right)
$$

where $\tau_{i}$ is the input delay, and $K$ is the time constant. It is important to note the parameters in (4) vary with the reference flow rate. The above first-order system takes into account the transport delay, in-stream storage phenomena and the dispersion of the flow (or wave attenuation) as it moves downstream. In this study the river reach model is simplified to a transport delay. As for storages described above, without this simplification, river reaches can still be described by a set linearized models by selecting a several operating points over the range of set-points. Once again, gain scheduling can be applied. With the above simplification, in discrete-time notation we have

$$
q_{\text {out }_{i}}[k+1]=q_{\text {in }_{i}}\left[k-\left\lceil\tau_{i} / T_{s}\right\rceil\right]
$$

\section{B. State-space representation}

We define a set of states that are underlying water levels with $x_{i, 0}[k]=y_{i}[k]$, or flows with $x_{i, 0}[k]=q_{i}[k]$. To deal with time delays associated with flows within the network, we introduce the states $x_{j, i}[k]=u_{j}[k-i]$ which implies that whenever the term $u_{j}[k-\tau]$ appears in the model equations we introduce the following auxiliary state equations

$$
\begin{aligned}
x_{j, 1}[k+1] & =u_{j}[k] \\
x_{j, i+1}[k+1] & =x_{j, i}[k], i=1, \ldots, \tau-1
\end{aligned}
$$

and the substitute $x_{j, \tau}[k]$ in all model equations where $u_{j}[k-$ $\tau$ ] appears [Wey03].

For the network in Fig. 1, the state equations for the first two storages and the last storage take on the form

$$
\begin{aligned}
x_{D, 0}[k+1] & =x_{D, 0}[k]+a_{D}\left(q_{D}[k]-u_{D}[k]\right) \\
x_{D, 1}[k+1] & =u_{D}[k] \\
x_{H, 0}[k+1] & =x_{H, 0}[k]+a_{H}\left(x_{D, 1}[k]+d_{M M}[k-1]\right. \\
& \left.+q_{I N}(k)-u_{H}[k]\right) \\
x_{H, 1}[k+1] & =u_{H}[k] \\
x_{H, i+1}[k+1] & =x_{H, i}[k], i=1, \ldots, 3 \\
\vdots & \\
x_{A, 0}[k+1] & =x_{A, 0}[k]+a_{A}\left(x_{1,10}[k]-u_{A}[k]-d_{A}[k]\right) \\
x_{A, 1}[k+1] & =u_{A}[k]
\end{aligned}
$$

The disturbances $q_{D}, d_{M M}, q_{I N}$ and $d_{A}$ are inflows or offtakes. All these disturbances are known in advance and their effects can be minimized through feedforward. Note that while the last control input $u_{A}[k]$ does not feed any storage within the network, it is still necessary to introduce the state $x_{A, 1}[k]$ if we are to control flow and gate movement of storage A.

Using the above set of equations as an example, the state space equation for the entire network can be generated and takes on the form

$$
\begin{aligned}
\mathbf{x}_{m}[k+1] & =A \mathbf{x}_{m}[k]+B \mathbf{u}_{m}[k]+w[k], \\
\mathbf{z}_{m}[k] & =C \mathbf{x}_{m}[k] .
\end{aligned}
$$

where $\mathbf{x}_{m}[k]=\left[x_{D, 0}[k], x_{D, 1}[k], x_{H, 0}[k], x_{H, 1}[k], \ldots\right.$, $\left.x_{H, 4}[k], \ldots, x_{A, 0}[k], x_{A, 1}[k]\right]^{T}, \quad \mathbf{u}_{m}[k]=\left[u_{D}[k], u_{H}[k]\right.$, $\left.\ldots, u_{A}[k]\right]^{T}$. Note that all state variables are accessible and so there is no need for an observer or estimator.

\section{MODEL PREDICTIVE CONTROL}

For the purpose of MPC design, the state-space model (6) is considered. The main control objectives are set-point tracking, minimizing energy consumption and disturbance rejection. As stated before, disturbance rejection can be 
achieved through feedforward. Denoting $H_{p}$ prediction horizon and $H_{u}$ control horizon, set-point tracking and minimizing energy consumption can be achieved by minimizing the following quadratic objective function at time $k$

$$
\begin{aligned}
J[k]= & \sum_{i=1}^{H_{p}}\left\|\mathbf{z}_{m}[k+i \mid k]-\mathbf{r}[k+i \mid k]\right\|_{Q[i]}^{2} \\
& +\sum_{i=0}^{H_{u}-1}\left\|\Delta \mathbf{u}_{m}[k+i \mid k]\right\|_{R[i]}^{2}
\end{aligned}
$$

subject to (6), where $\mathbf{r}$ is a filtered version of set-point signals, $\Delta \mathbf{u}_{m}[i]:=\mathbf{u}_{m}[i]-\mathbf{u}_{m}[i-1]$.

As introduced in Section I-B, river operation is subject to many constraints. Both hard constraints and soft constraints are considered here.

\section{Hard constraint}

Hard constraints are those which cannot be violated, for example, positive water level and flow rate, and bounds on velocity of gate movement,

$$
\mathbf{x}>0, \quad \mathbf{u}>0, \quad\left|\Delta \mathbf{u}_{m}\right|<\epsilon_{u}
$$

Note that hard constraints should be carefully chosen as too many hard constraints would bring the underlying optimization problem infeasible.

\section{Soft constraint}

Soft constraints can be violated, but only for a very short period, to prevent the overall optimization problem infeasible, for example, the upper and lower bounds on outputs,

$$
\underline{\epsilon}_{z}<\mathbf{z}_{m}<\bar{\epsilon}_{z}
$$

This is done by introducing slack variables, however, which means more computation cost than hard constraints as a compromise.

Now, a typical optimization problem at time $k$ can be formulated as follows,

$$
\text { minimize } \mathrm{J}[\mathrm{k}] \text {, subject to (6) and underlying constraints, }
$$

where $J[k]$ is defined in equation (7). Once the optimal solution $\left\{\Delta \mathbf{u}[k \mid k], \Delta \mathbf{u}[k+1 \mid k], \cdots, \Delta \mathbf{u}\left[k+H_{u}-1 \mid k\right]\right\}$ to the optimization problem in (8) is obtained, only the first one $\Delta \mathbf{u}[k \mid k]$ is used to calculate the control action at time $k, \mathbf{u}[k]=\Delta \mathbf{u}[k \mid k]+\mathbf{u}[k-1]$.

\section{EXPERIMENTAL RESULTS}

The following section outlines simulation results obtained using BasinCad, a computer aided design software tool for simulating river basin networks. $H_{p}=10$ and $H_{u}=5$ are used in the following examples.

\section{A. Weighting matrices selection}

For this example we set $\mathbf{Q}=I$ and $\mathbf{R}=\sigma I$ where $I$ is the identity matrix with appropriate dimension and $\sigma$ is the coefficient in weighting matrix $\mathbf{R}$ which we need to select to achieve better performance. A unit step input disturbance with amplitude of $1 \mathrm{GL} /$ day was introduced at $q_{D}$. Figures 2, 3(a), and 3(b) show rate of change of flow rate, storage water level and flow rate transients for different $\sigma^{\prime} s$. It is assumed that $250 \mathrm{ML} /$ day is the maximum permissible rate of change of flow rate. Note that the system should try to operate as close to the constraint limits as possible. For that purpose, using Figure 2, we select $\sigma=10^{-10}$ for the results that follow.

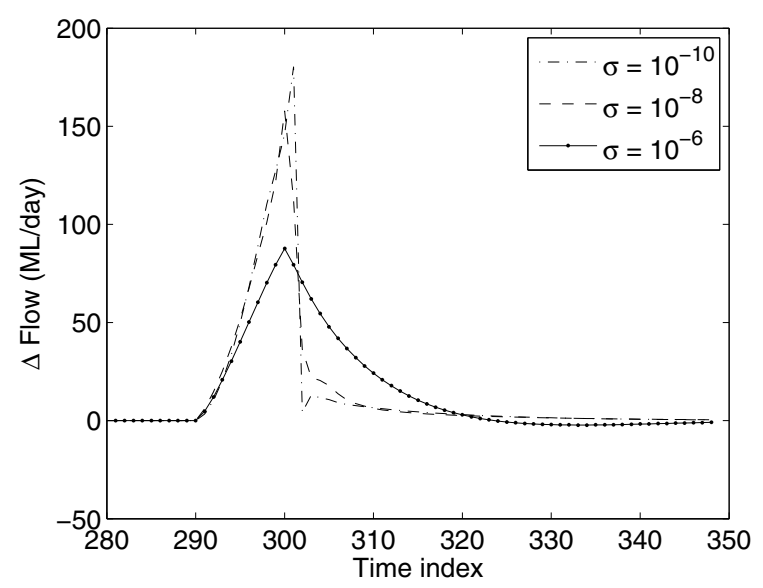

Fig. 2. Rate of change of flow-rate transients at storage $\mathrm{H}$ for varying $\sigma$ in response to disturbance flow $q_{D}$.

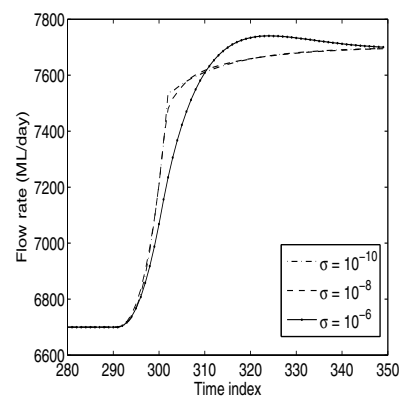

(a) Flow rate transients

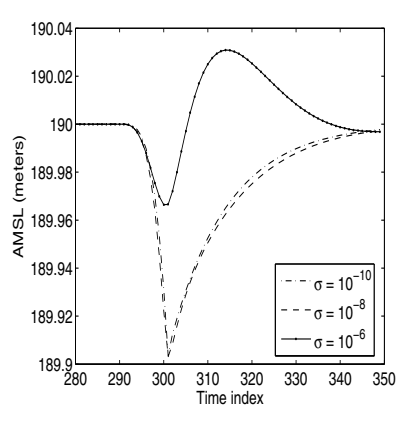

(b) Water level transients
Fig. 3. Water level and flow transients at storage $\mathrm{H}$ for varying $\sigma$ in response to disturbance flow $q_{D}$.

\section{B. Disturbance rejection}

Figure 4 illustrates the effect of upstream disturbances and compares the performance of the MPC controller scheme. In this example a step change at $q_{D}$ of $1 \mathrm{GL} /$ day is introduced at time index 300. Figures 4(a) and 4(b) illustrate the corresponding water level and flow rate transients at Dams $\mathrm{H}$ and A for the MPC controller. The results clearly indicate firstly the pre-release of water to accommodate the disturbance 
inflow. This is apparent from the rise in water levels and flow rates before time index 300 at Dam $\mathrm{H}$. Secondly, the integral action inherent in this system smoothes out the transients as we move further downstream Dam A.
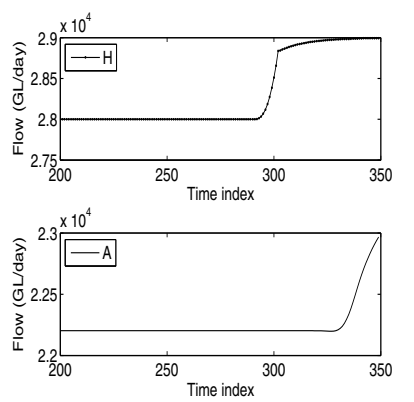

(a) Flow response

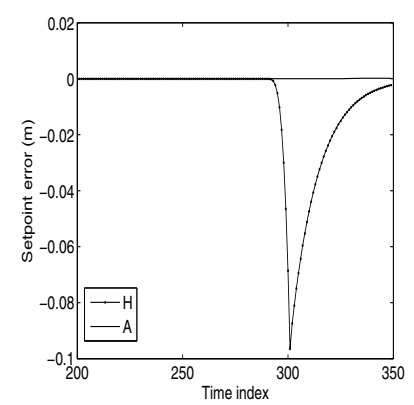

(b) Level response

Fig. 4. Disturbance rejection at storages $\mathrm{A}$ and $\mathrm{H}$ in response to disturbance flow $q_{D}$.

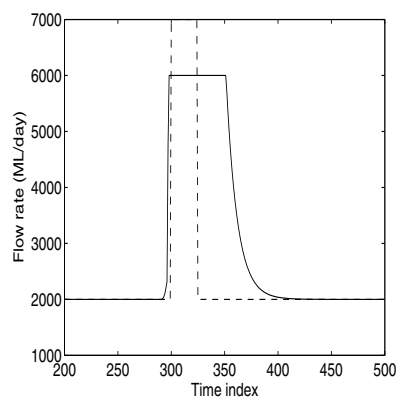

(a) Flow response at Dam D

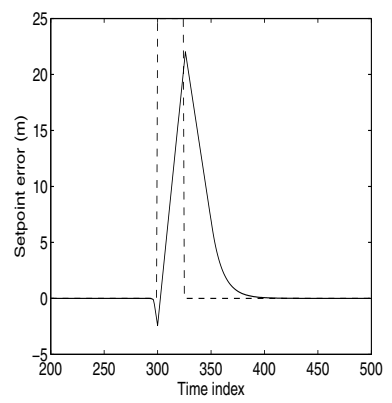

(b) Level response at Dam D
Fig. 5. Flood mitigation using MPC.

\section{Flood mitigation}

An important function for a controller is water pre-release for flood mitigation. This is demonstrated by generating a pulse disturbance of amplitude $10 \mathrm{GL} /$ day over 25 days resulting a total volume of 250GL. The immediate downstream Dam D has an output flow constraint set at 6GL/day and a maximum water level of $486 \mathrm{~m}$. A successful control strategy must incorporate pre-release to accommodate the outflow constraint and maximum water level constraint. Figure 5 illustrates the water level and outflow from Dam D in response to the disturbance indicated by the dashed line. Here the water level setpoint at Dam D is $462 \mathrm{~m}$. The key point to note is the mandatory pre-release which is evident in Figure 5(a) between time indices 290 and 300 .

\section{Robustness test}

Since BCad software has independent control module and simulator module, we can assign different sets of parameters of the whole network for the former and the latter module. By doing this, we can carry out simulations to demonstrate the case where the signals or parameters at the simulator module are different from those known to the controller module.
1) Disturbance mismatch: Figure 6 shows the effect of disturbance mismatch on the water level at Dam D when the controller design is based on one set of disturbance flows (incorrect $q_{D}$ ) while the simulator module is run using a different set of disturbance flows (correct $q_{D}$ ). The poor quality of water level control for the unmatched case can be attributed to the failure of the feedforward component of the controller as it tries to carry out disturbance rejection with the wrong set of disturbance flows.
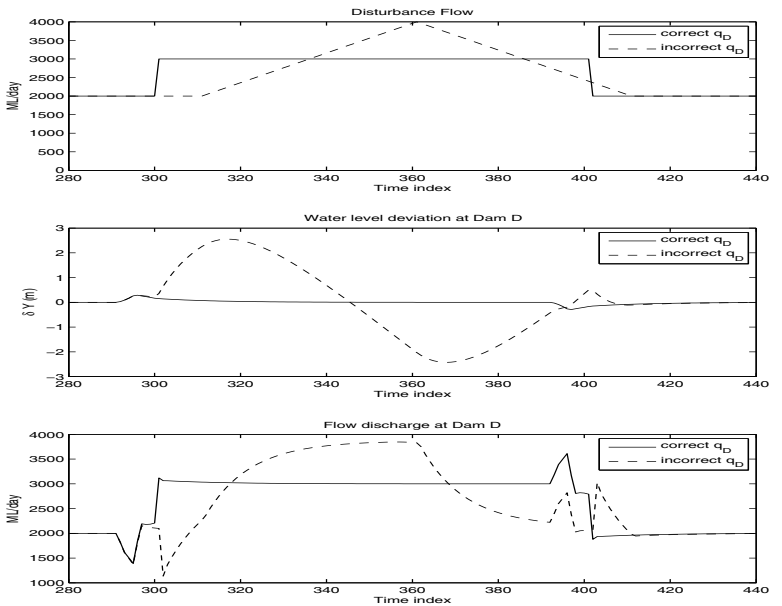

Fig. 6. Effect of disturbance on water level control when an incorrect disturbance is used in place of the correct one.

2) Dam surface area mismatch: Figure 7 shows the results for the cases where the surface area of Dam D is over-estimated and under-estimated by $50 \%$ respectively. For the former case, the controller still thinks that the dam is $50 \%$ larger and hence control inputs are also larger. The water level therefore fluctuates more wildly. The opposite is observed for the latter case. The water level will rise and fall at a much slower pace due to small values of control inputs. Moreover, the initial transient is slower to reach the set points. The effect at Dam A is negligible compared to that at Dam D. Therefore errors in dam parameters seem to be localized at the affected dam.

3) Delay mismatch: It is well known that control of flow networks with transport lags require accurate knowledge of time delays. As indicated in the previous discussion, the transport delays in the link element change with flow rate. In this paper we assume that these delays are constant, however this not always the case. Figure 8 shows the effect of delay mismatch. The delay in the link between Dam H and Dam Y used in the simulator is 4 days while that used in the controller is 10 days. The error in delays will cause change in dimensions of system matrices. The effect is more pronounced in Dam Y than Dam A. This is due to the close proximity of Dam Y to the link. The disturbance rejection fails during the period of 6 days around the transitions. This is because the feedforward part of the controller expects the 

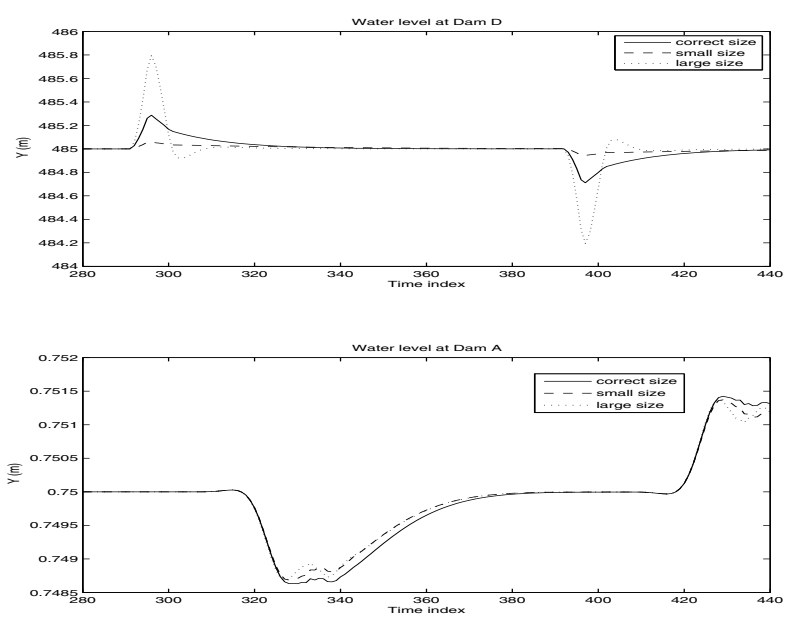

Fig. 7. Effect of dam surface area mismatch at Dams D and A. Surface area of Dam D is over-estimated and under-estimated by $50 \%$ of the correct value respectively.

disturbance 6 days later. The feedback part of the controller continues to function properly and the water levels eventually converge to the set points. Delay mismatch can be said to manifest itself as disturbance mismatch.
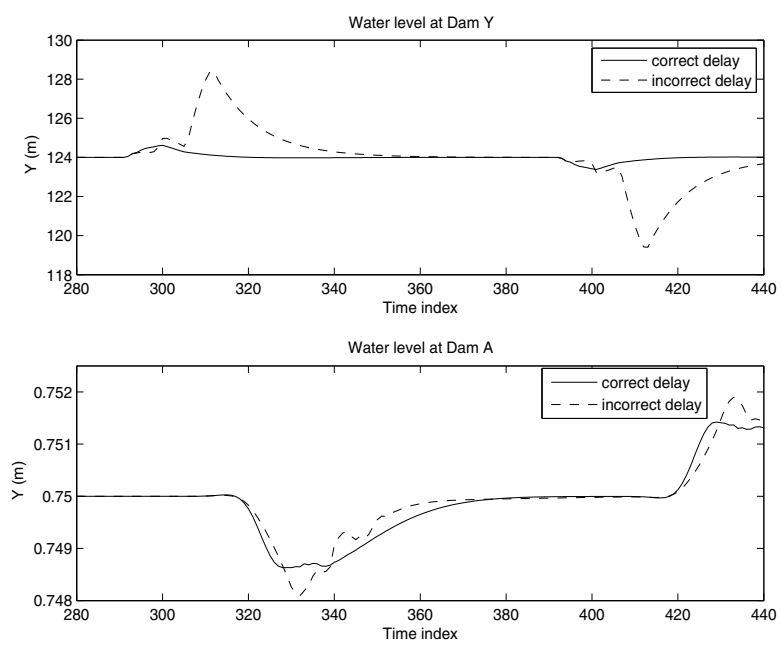

Fig. 8. Effect of delay mismatch at Dams Y (top) and A (bottom). Delay between Dams $\mathrm{H}$ and $\mathrm{Y}$ is over-estimated from 4 days to 10 days.

\section{CONCLUSIONS AND FURTHER WORK}

A systematic framework of modeling and controlling river basin networks is introduced in this paper using simple linear models and model predictive control scheme. The effects of disturbances, constraints, and sensitivity to different sets of parameter mismatch are investigated. Future research work will focus on the incorporation of nonlinear dynamics, forecasting module, distributed control and water quality control.

\section{REFERENCES}

[BB09] R. J. Bridgart and M. Bethune. Development of RiverOperator: a tool to support operational management of river systems. In 18th World IMACS/MODSIM Congress, pages 3782-3788, 2009.

[BWdMB08] T. B. Blanco, P. Willems, B. de Moor, and J. Berlamont. Flood prevention of the demer using model predictive control. In the 17th IFAC World Congress, pages 3629-3934, Seoul, Korea, 2008.

[Cho88] V. T. Chow. Open-Channel Hydraulics. McGraw-Hill, New York, 1988.

$\left[\mathrm{ELM}^{+}{ }^{11}\right.$ R. Evans, L. Li, I. Mareels, N. Okello, M. Pham, W. Qiu, and S. K. Saleem. Real-time optimal control of river basin networks. In Control and Identification Challenges in the Management of Water Networks. Invited book chapter, Springer-Verlag, 2011. To be published.

[JH93] A. J. Jakeman and G. M. Horngerger. How much complexity is warranted in a rainfall-runoff model. Water Resources Research, 29:2637-2649, 1993.

[Lab04] J. W. Labadie. Optimal operation of multireservoir systems: State-of-the-art review. Journal of Water Resources Planning and Management, 130:93-111, 2004.

[LF06] X. Litrico and V. Fromion. $H_{\infty}$ control of an irrigation canal pool with a mixed control politics. IEEE Transactions on Control Systems Technology, 14(1):99-111, 2006.

[LG99] X. Litrico and D. Georges. Robust continuous-time and discrete-time flow control of a dam-river system: (I) modellling. Applied Mathematical Modelling, 23:809-827, 1999.

[Mac02] J. M. Maciejowski. Predictive Control with Constraints. Prentice Hall, London, 2002.

[Mar09] I. Mareels. Abandoning babylonian principles to rebuild irrigation infrastructure. ATSE Focus, issue 157, August/September 2009.

[MDB06a] MDBC. Murray-Darling Basin Water Resources Fact Sheet. Murray-Darling Basin Commission, 2006.

[MDB06b] MDBC. Running the river. Murray-Darling Basin Commission, 2006.

[MRRS00] D. Q. Mayne, J. B. Rawlings, C. V. Rao, and P. O. M. Scokaert. Constrained model predictive control: Stability and optimality. Automatica, 36(6):789-814, 2000.

$\left[\mathrm{MWO}^{+} 05\right]$ I. Mareels, E. Weyer, S. K. Ooi, M. Cantoni, Y. Li, and G. Nair. Systems engineering for irrigation systems: successes and challenges. Annual Reviews in Control, 29(2):191-204, 2005.

[NvOKdS09] R. R. Negenborn, P. J. van Overloop, T. Keviczky, and B. de Schutter. Distributed model predictive control of irrigation canals. Networks and Heterogeneous Media, 4(4):359380, 2009.

[Pap84] M. Papageorgiou. Optimal control of generalized flow networks. In P. Thoft-Christensen, editor, System Modelling and Optimization, volume 59 of Lecture Notes in Control and Information Sciences, pages 373-382. Springer, Berlin / Heidelberg, 1984.

[SA90] J. S. Shamma and M. Athans. Analysis of gain scheduled control for nonlinear plants. IEEE Transactions on Automatic Control, 35(8):898-907, 1990.

[vO06] P. J. van Overloop. Model Predictive Control of Open Water Systems. PhD thesis, Delft University of Technology, Delft, The Netherlands, 2006.

[Wey01] E. Weyer. System identification of an open water channel. Control Engineering Practice, 9(12):1289-1299, 2001.

[Wey02] E. Weyer. Decentralized PI control of an open water channel. In 5th Triennial World Congress, Barcelona, Spain, 2002. IFAC.

[Wey03] E. Weyer. LQ control of an irrigation channel. In 42nd IEEE Conference on Decision and Control, volume 1, pages 750-755, 2003.

[WM73] C. B. Winn and J. B. Moore. The application of optimal linear regulator theory to a problem in water pollution. IEEE Transactions on Systems, Man, and Cybernetics, 3:450-455, 1973.

[You98] P. C. Young. Data-based mechanistic modelling of environmental, ecological, economic and engineering systems. Environmental Modelling and Software, 13:105-122, 1998. 